\title{
Myocardial infarct size and cardiac performance at exercise soon after myocardial infarction ${ }^{\star}$
}

\author{
PEER GRANDE, ASGER PEDERSEN \\ From the Department of Cardiology and Department of Clinical Chemistry, Glostrup Hospital, University of \\ Copenhagen, Denmark
}

SUMMARY Infarct size was estimated from serial serum CK MB measurements in a series of $101 \stackrel{ \pm}{ \pm}$ patients admitted less than 15 hours after the first acute myocardial infarction. A maximal symptom $\overrightarrow{.}$ limited exercise test comprising impedance measurements for the estimation of stroke volume at rest and at different levels of exercise was performed early after admission by 26 patients.

There was a slight, though not significant, negative correlation between infarct size and physical $\vec{c}$ capability as measured by the duration of work. The rise in systolic blood pressure during exercise $\stackrel{\Xi}{5}$ showed a significantly negative and the increase in heart rate a significantly positive correlation to infarct size. This suggests that the rise in blood pressure, which is less in patients with the larger ${ }^{2}$ infarcts, is compensated by an increase in heart rate, so that the same maximum of cardiac perfor- $\vec{\oplus}$ mance and myocardial oxygen consumption is reached. The increase in cardiac stroke volume ${ }^{\infty}$ during exercise was negatively correlated with infarct size. Stroke volume only increased during lower levels of exercise; the increase in cardiac output at higher levels of exercise was achieved entirely by an increase in heart rate. The magnitude of ST segment elevation during exercise showed a significantly positive correlation with infarct size, whereas the occurrence of arrhythmias during exercise was independent of it.

The extent of myocardial injury in acute myocardial infarction and its possible impact on the subsequent course of the disease have attracted much attention in recent years. Hence, in vivo estimation of infarct size has become of importance, and various methods of estimation have been applied. ${ }^{1}$

Some studies after acute myocardial infarction have investigated the relation between the estimated infarct size and cardiac performance, measured either as the ejection fraction of the left ventricle by radionucleocardiography, ${ }^{23}$ or through the pulmonary haemodynamics at right heart catheterisation. ${ }^{4}$ The exercise test provides information about several aspects of cardiac performance, and is applicable also during the first weeks after acute mycocardial infarction. There are no reports, however, of a comparison between the results of exercise tests and the estimated infarct size in a large series of patients.

^This study was supported by grants from the Danish Heart Foundation, A/S Nordisk Gjenforsikrings Selskabs Jubilæumsfond, Købmand i Odense Johann og Hanne Weimann f. Seedorffs Legat, and Carl J. Beckers Fond. Peer Grande is a recipient of a research fellowship from the University of Copenhagen.

Accepted for publication 28 July 1981
Exercise tests three weeks after acute myocardial infarction have been routine at our unit since January? 1972.5 Moreover, in recent years estimation of the infarct size from serum levels of creatine kinase isoen- $\frac{0}{7}$ zyme MB (CK MB) has shown a satisfactory correla-tion with anatomical measurements taken at nec- 3 . ropsy. ${ }^{6}$ In this study the estimated infarct size and the results of exercise tests, usually carried out three weeks after the onset of acute myocardial infarction, 을 were compared in a series of patients with the first infarct.

\section{Patients}

We investigated a series of patients with acute $\tilde{\omega}_{\mathrm{W}}$ myocardial infarction who met the following criteria:0 no previous history of acute myocardial infarction duration of infarct less than 15 hours, sufficient data for estimating the infarct size from serial enzymes? measurements, and ability to do an exercise testo according to our standard procedure. The patientso were taken out of all admissions to our coronary cares unit during the course of 12 months. As all cases of suspected acute myocardial infarction without excep- 
tion are admitted to the coronary care unit and as deliberate treatment of such infarction in the home is rare in Denmark, we believe that our population is representative of virtually all those who survive an acute myocardial infarction long enough to reach hospital.

Between 1 June 1978 and 1 June 1979 the coronary care unit received 393 patients with acute myocardial infarction, defined according to the criteria given below. The duration of the infarct was less than 15 hours in 345, and sufficient data for calculating the infarct size from determinations of the serum CK MB were available in 321 . Acute myocardial infarction had occurred for the first time in 225 patients. Twenty-eight died during admission and 197 were discharged from the coronary care unit after an observation period of slightly less than three weeks. Out of these 197 patients, 96 were unable to perform the exercise test for the reasons stated in Table 1. These

Table 1 Reasons for non-performance of exercise test soon after acute myocardial infarction

\begin{tabular}{lc}
\hline Reasons & $N o$. \\
\hline Heart failure, pronounced & 8 \\
Hypertension, pronounced & 4 \\
Angina pectoris, severe and persistent & 4 \\
Atrioventricular block, treated with temporary pacemaker & 3 \\
Intermittent bundle-branch block & 2 \\
Postinfarction syndrome & 1 \\
Pulmonary conditions (bronchial asthma, pulm. embolism, & \\
pneumonectomy, etc.) & 4 \\
Intermittent claudication & 9 \\
Gangrene of foot & 1 \\
Joint disorders & 9 \\
Paralysis of extremity & 10 \\
Urethral catheter & 2 \\
Blindness & 1 \\
Other medical disorders (jaundice, dyspepsia, epilepsy) & 4 \\
Age and/or poor general condition & 20 \\
Refusal to participate & 10 \\
Unable to bicycle & 2 \\
Lack of laboratory staff & 2 \\
\hline Total & 96 \\
\hline
\end{tabular}

were 64 men between 48 and 93 years of age (mean 67.2 years) and 32 women between 46 and 83 years of age (mean 68.1 years).

Thus, our study group comprises 101 patients who survived the first acute myocardial infarction, who were able to do the exercise test, and in whom the infarct size could be estimated. There were 88 men between 23 and 81 years of age (mean 54.6 years) and 13 women between 42 and 73 years of age (mean 56.2 years). As was to be expected, there was a predominance of men and of somewhat younger people who were able to perform the test.

\section{Methods}

Acute myocardial infarction was defined by the occurrence of typical clinical symptoms and/or typical evolution of electrocardiographic abnormalities, when confirmed by a temporary rise in serum CK MB levels. ${ }^{7}$ Electrocardiograms in six praecordial and three extremity leads were recorded daily. Blood samples were drawn on admission, and approximately every eight hours thereafter until the CK MB values were normal. CK was analysed according to the Scandinavian recommended methods, and CK MB was determined by electrophoresis on agarose gel followed by fluorescence scanning. ${ }^{8}$ The interassay variation of the technique is below $10 \%$ in the lower and below $5 \%$ in the higher range of activity.

Infarct size was calculated from the serum CK MB values, according to a method described elsewhere, ${ }^{9}$ and expressed in $\mathrm{U} /$.

Eighty-six patients performed the exercise test according to the standard procedure of the unit, that is during the third week after the onset of the acute myocardial infarct and a few days before discharge from hospital. The test was postponed in the case of 15 patients: in four to week four, in five to week five, in three to week six, in one to week seven, and in two to week eight. The reasons were reinfarction or progression of the first infarction on the fourth day (one patient), complicating thrombophlebitis (one patient), and lack of laboratory staff over the Christmas holidays, etc. (13 patients).

At the beginning of the test the patients rested supine for at least 10 minutes, while self-adhesive electrocardiograph electrodes were placed in the deltoid regions, in the groins, and on the chest positions at V1-V4-V6, and a blood pressure cuff was placed round the upper arm. ${ }^{5}$

During the test the stroke volume in some of the patients could be calculated repeatedly from measurements of variations in thoracic impedance: two mylar bands were placed around the neck and two around the lower thorax, and a microphone was placed in the aortic area, all connected to the IFM/ Minnesota Impedance Cardiograph Model 304 A.

The graded exercise was done on a bicycle ergometer with electronically controlled braking (SiemensElema A.M. 368), the load being increased in stages from 50 to 100 to 150 watts, remaining at each stage for six minutes in order to ensure a steady state. The aim was a maximal, symptom limited performance, and the patient was encouraged to proceed until he reached the limit set by such physiological symptoms as fatigue and dyspnoea. The reasons for stopping the exercise were pathological symptoms, such as anginal or leg pain; objective signs, such as pronounced and rapidly increasing changes in the ST segment, extrasystoles, especially when multiform or appearing in series or close to the preceding $\mathrm{T}$ wave; and either a rapid rise in blood pressure to very high values, or the absence of a rise in blood pressure. 
Electrocardiograms in three extremity leads and three praecordial leads and heart rate were recorded continuously, and blood pressure was measured every minute throughout the test. All measurements were continued through the following periods: supine at rest for at least three minutes, sitting on the bicycle at rest for three minutes, sitting on the bicycle during exercise, and sitting on the bicycle at rest for 10 minutes after exercise.

In the electrocardiograms any ST segment deviation from the level of the PR segment to the TP segment was measured 0.06 second after the nadir of the last wave of the QRS complex.

Rhythm disturbances were assessed before exercise (at least three minutes supine plus three minutes sitting on the bicycle) and from the continuous electrocardiographic recording during the entire period of exercise and for 10 minutes after.

In those patients prepared for impedance measurements, the $\mathrm{dZ} / \mathrm{dt}$ curve was recorded by an electrocardiograph writer (Mingograph 34, Elema), simultaneously with one unipolar chest lead of the electrocardiograph, and a phonocardiogram from the aortic area. The stroke volume was calculated according to Kubicek et al., ${ }^{10}$ through 10 cardiac cycles at the end of the following periods: supine at rest, sitting at rest, at each increase in exercise load, and 10 minutes after cessation of exercise.

Statistical calculations were made by the rank test for unpaired data (Mann-Whitney test). The $t$ test was used for testing if the coefficients of correlation were different from zero.

\section{Results}

Exercise was discontinued in the 101 patients for the reasons given in Fig. 1. Two-thirds, or 67 patients, were able to continue exercise until extreme fatigue and dyspnoea set in, three stopped mainly because of nervousness, and 17 were stopped by anginal pain. In only 14 cases was the exercise interrupted by the investigator: in four because of frequent, but always single ventricular extrasystoles; in three because of the development of very pronounced ST deviation; in one because of an insufficient rise in blood pressure; and in six because of a rapid and unacceptably high rise in blood pressure. In all cases, the rhythm disturbance or electrocardiographic abnormalities disappeared or the blood pressure approached initial values within a few minutes after stopping exercise. More severe arrhythmias, persistent electrocardiographic abnormalities, signs of reinfarction, or other serious complications did not occur.

Fig. 1 also shows the performance of work which ranged from two minutes of exercise at 50 watts to 21 minutes at the phased increase in load from 50 to 100

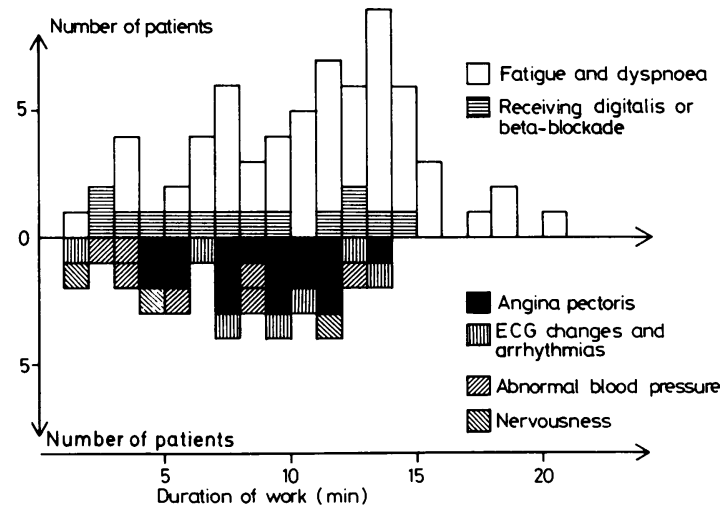

Fig. 1 Performance during exercise testing on a bicycle ergometer at 50 watt for 6 minutes + 100 watt for 6 minutes + 150 watt for 6 minutes, etc., in 101 patients, 15 to 53 days (median 17 days) after the onset of their first acute myocardial infarct. Above the horizontal line: the performance of 67 patients continuing exercise until intolerable fatigue and dyspnoea. Below the line: 34 patients who stopped exercise for other reasons.

to 150 to 200 watts, remaining at each load for six minutes. Among the 67 patients who continued exercise until the physiological limit set by fatigue and dyspnoea, the median exercise duration was 12.0 minutes (quartiles 8 and 14 minutes). Within this group of 67 the 14 who received digitalis and/or a beta-blocking agent had a significantly lower capacity of work (median 8.5 minutes) when compared with the rest (median 12.0 minutes, $\mathrm{p}=0.03$ ).

Patients whose exercise was stopped by the investigator, because of electrocardiographic abnormalities, or arrhythmias, or abnormal blood pressure response, and those who stopped because of anginal pain, had a significantly lower capacity of work than the patients who stopped exercise only because of fatigue and dyspnoea $(p=0.03$, and $p=0.01)$.

Table 2 shows the relation between the estimated infarct size and duration of work for the 53 patients who had not had digitalis or beta-blockers and who continued exercise until the limit of physiological fatigue and dyspnoea. The median infarct size in this group was $756 \mathrm{U} / 1$, range 60 to $2973 \mathrm{U} /$, corresponding roughly to between $3 \mathrm{~g}$ and $140 \mathrm{~g}$ of infarcted tissue. ${ }^{6}$ There was only a slight, statistically

Table 2 Relation between estimated infarct size, duration of work, and age $(n=53)$

\begin{tabular}{llll}
\hline & $r$ & $S E E \%$ & $p$ \\
\hline Infarct size vs. duration of work & -0.15 & 30 & 0.25 \\
Age vs. infarct size & -0.36 & 26 & 0.004 \\
Age vs. duration of work & -0.42 & 32 & 0.002 \\
\hline
\end{tabular}


insignificant, negative correlation between infarct size and duration of work. We found, however, a significantly negative correlation between age and infarct size. As there was also a strong negative correlation between age and work performance, the influence of age tends to veil the negative correlation between infarct size and work performance.

Table 3 shows the relation between the estimated infarct size and the response in systolic blood pressure

Table 3 Relation between estimated infarct size and response to exercise test

\begin{tabular}{|c|c|c|c|c|c|}
\hline & & \multicolumn{4}{|c|}{ Infarct size $(C K M B U / l)$} \\
\hline & & No. & $r$ & $S E E \%$ & $p$ \\
\hline \multirow{2}{*}{$\begin{array}{l}\text { Systolic blood } \\
\text { pressure }\end{array}$} & Max. value & 53 & -0.32 & 9 & 0.02 \\
\hline & Rise $^{\star}$ & 48 & -0.46 & 24 & 0.01 \\
\hline \multirow{2}{*}{ Heart rate } & Max. value & 53 & 0.39 & 11 & 0.00 \\
\hline & Rise $^{\star}$ & 48 & 0.39 & 30 & 0.00 \\
\hline
\end{tabular}

$\star$ Expressed as the difference between the steady state value of work load $100 \mathrm{~W}$ and the resting value, both while sitting on the bicycle.

and heart rate during exercise. A significantly negative correlation was found between the infarct size and the rise in systolic blood pressure, whether the latter was understood as the absolute maximum reached in 53 patients, or as the rise from sitting at rest to exercising at 100 watts in 48 patients. A significantly positive correlation was found between the infarct size and the increase in heart rate, as regards the absolute maximal values and the rise from resting to maximal exercise values. As these two correlations cancelled each other, the pressure rate product showed no significant correlation to infarct size.

In Fig. 2 the estimated infarct size is related to the maximal increase in stroke volume index and cardiac index, as estimated from the variations in thoracic impedance during exercise. The maximal increase in stroke volume index and in heart rate was measured as the difference between the maximal value during exercise and the value measured when sitting on the bicycle just before starting exercise. The resting values of the stroke index and heart rate showed no significant correlation with the infarct size. There was, however, a significantly negative correlation between the infarct size and the rise in stroke index $(p=0.01)$, and in cardiac index $(p=0.03)$. As regards the increase in stroke index, the range of results was broad in the patients with the smallest infarcts whereas it was low in those with larger infarcts. We did not find the combination of a large infarct size and a high increase in stroke volume during exercise. Stroke index did not increase at all in four patients
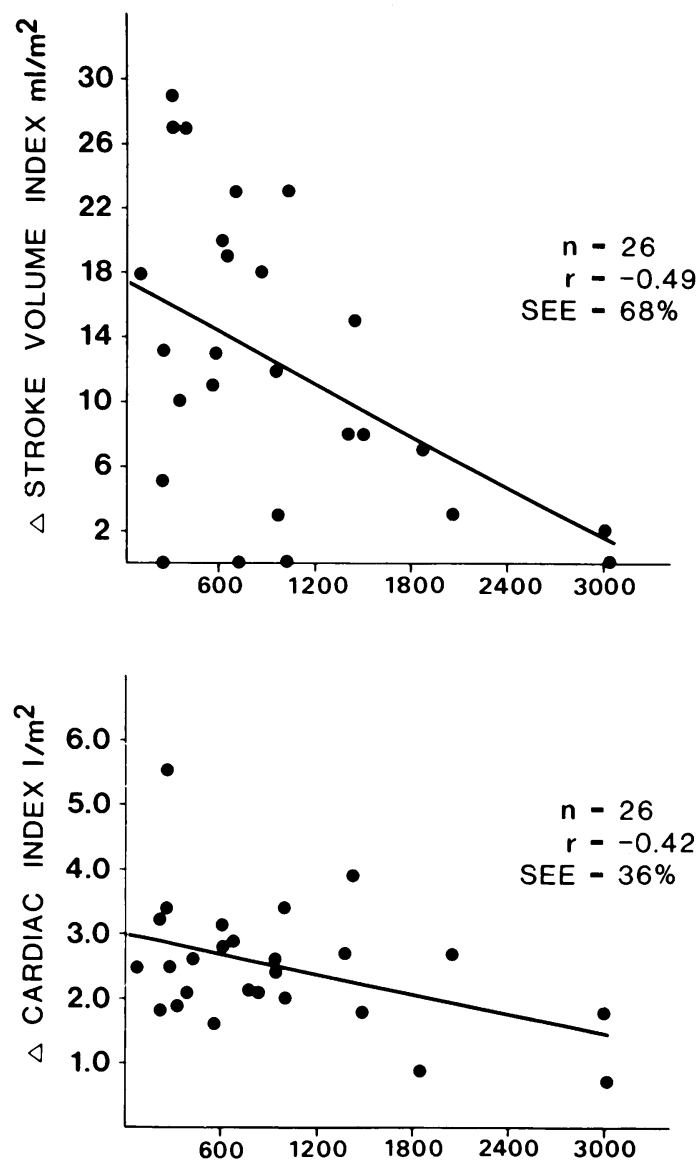

ESTIMATED MYOCARDIAL INFARCT SIZE

(COMPARTMENT MODEL CK MB U/I)

Fig. 2 Relation between the estimated myocardial infarct size and increase in stroke volume index (above, $p=0.01$ ) and in cardiac index (below, $p=0.03$ ) during exercise calculated from impedance measurements.

during exercise, but, because of increased heart rate, the cardiac index rose in all 26 patients.

Fig. 3 shows the values in 26 patients of stroke volume, heart rate, cardiac output, and rate-pressure product at five points of the exercise test: resting supine, resting sitting on the bicycle, after six minutes work at 50 watts, after 12 minutes work at 50 to 100 watts (in the 23 patients who were able to continue 12 minutes of work), and 10 minutes after cessation of exercise still sitting on the bicycle. At rest before exercise the transition from supine to sitting position was followed by a considerable decrease in the stroke volume and only a small increase in heart rate, and consequently a distinct drop in the calculated cardiac output. During the first six minutes of exercise, 


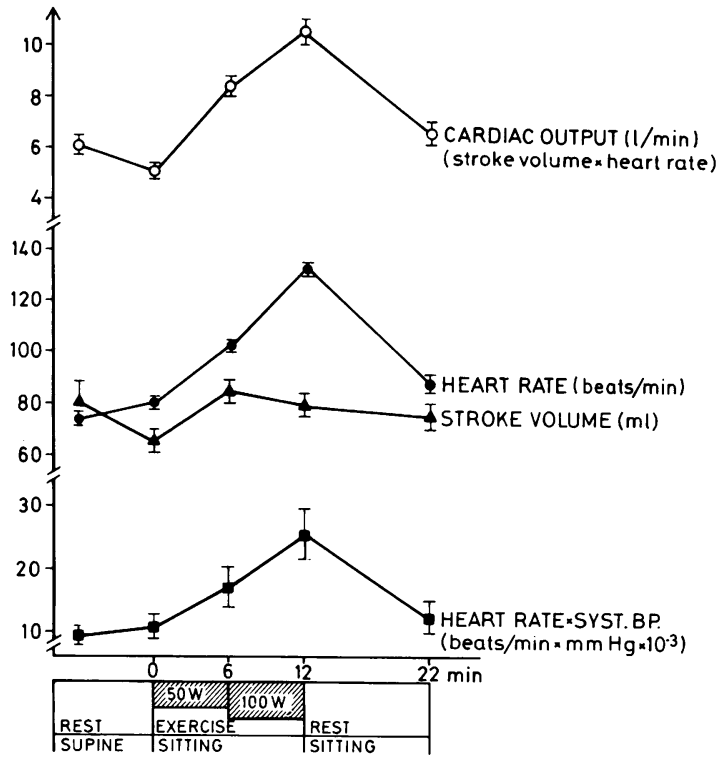

Fig. 3 Mean value \pm standard error of the mean of stroke volume, heart rate, cardiac output (as the product of stroke volime and heart rate), and the product of heart rate and systolic blood pressure, all measured at the same five points of the exercise test. See text.

stroke volume and heart rate rose and cardiac output was much increased. At the higher levels of exercise up to the maximal performance, stroke volume remained constant or even decreased again, so that the further rise in cardiac output was caused only by increased heart rate. Comparison with the infarct size calculated in each patient showed a significantly negative correlation between that and the rise in stroke volume during the first level of exercise $(r=-0.33$, $p=0.03$ ), but no correlation between infarct size and the variation in stroke volume after the first six minutes of exercise $(r=-0.11, \mathrm{NS})$. The pressurerate product was increased two-and-a-half times on average during exercise, but, as mentioned, this increase showed no correlation to the infarct size.

Table 4 shows the relation between the infarct size and deviations of the ST segment during exercise in the 53 patients who continued exercising until stopped by dyspnoea and fatigue. No correlation could be shown between the infarct size and depression of ST segment during exercise, but a barely significant positive correlation was found between the infarct size and ST elevation during exercise. The median infarct size was $730 \mathrm{U} / \mathrm{f}$ for those without ST elevation, $784 \mathrm{U} / \mathrm{l}$ in 12 patients with ST elevation of 0.1 or $0.2 \mathrm{mV}$, and $2210 \mathrm{U} / \mathrm{l}$ in six patients with ST elevation larger than $0.2 \mathrm{mV}$. The difference between the two subgroups was not statistically significant. Among the 18 patients
Table 4 Estimated myocardial infarct size and changes in $S T$ segment during exercise (see text)

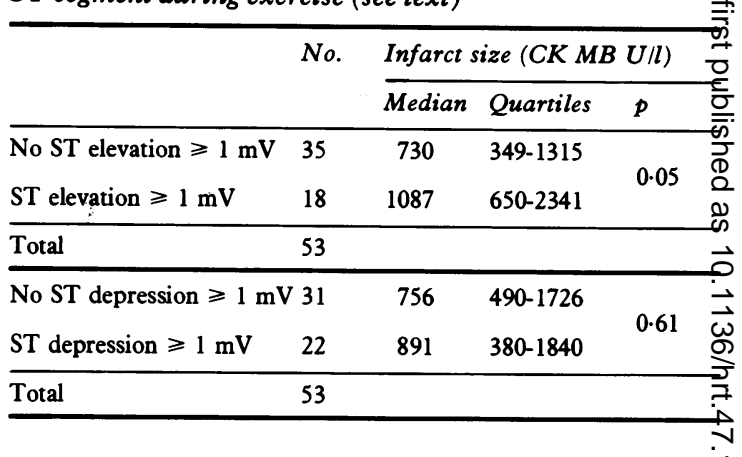

with ST elevation during exercise, 13 had an anteriois infarction and five an inferior infarction.

Of the 17 patients who were stopped by angina? pectoris during the exercise test, an ST deviation was absent in two but present in 14 , and $0.2 \mathrm{mV}$ or more in 10 of these, the deviation being an ST depression in nine and an ST elevation in five. The median infarct size was significantly smaller in the 17 patients wheno compared with the 53 patients who stopped becausê of fatigue and dyspnoea (median $363 \mathrm{U} / 1$, versus $756^{\circ}$ $\mathrm{U} / \mathrm{l}, \mathrm{p}=0.03$ ).

During observation before exercise, rhythm distur bances were present in 13 of the 101 patients, namelyp ventricular extrasystoles in nine, supraventricularo extrasytoles in two, and atrial fibrillation in two Arrhythmias increased in all 13 patients and occurredP in another 41 patients during exercise. Thus, 54 patients presented arrhythmias during exercise, in 44 of them as ventricular ectopic beats. There was, how ever, no significant connection between the infarcto size and the manifestation or exacerbation of either ventricular or supraventricular arrhythmias duringe the exercise test.

\section{Discussion}

The size of a myocardial infarct, as calculated fromp serial determinations of blood levels of the cardio specific enzymes, has shown a good correlation with the nitro-BT stained infarct size measured at nec=ropsy. ${ }^{46}$ The calculated infarct size has been found to be of negative importance in the functionat classification of patients after acute myocardial infarc tion $^{11}$ and in their life expectancy. ${ }^{12}$

Several studies have shown exercise testing three weeks after acute myocardial infarction to be safe? The test also provides important information on the capability of the diseased heart, and on the short-term? and long-term prognosis after an acute infarction Thus the increase in stroke volume, systolic blook pressure and oxygen consumption has been found tQ 
be smaller and the rise in heart rate higher in patients with a previous history of acute myocardial infarction than in those with ischaemic heart disease without such infarction ${ }^{13}$ and than in normal subjects. ${ }^{14}$ Moreover, the occurrence of ST depression and anginal pain and a smaller rise in the pressure-rate product at exercise have been found to indicate a relatively poor prognosis. ${ }^{15} 16$ In another series, impaired cardiac pump function and clinical signs of congestive heart failure were clear signs of a deteriorated prognosis after acute infarction. ${ }^{17}$

Reports on the correlation between estimated size of the myocardial injury and cardiac performance soon after an acute myocardial infarction have been few. Paine et al. ${ }^{18}$ investigated 100 patients several months after infarction with an equal share of one-, two-, and three-vessel disease at coronary arteriography. They found a significantly positive correlation between duration of exercise and left ventricular ejection fraction at angiography, a significantly negative correlation between duration of exercise and scar size estimated at angiography, and a larger ST segment elevation during exercise in patients with lower ejection fraction and larger angiographic scar size. In 50 patients with acute myocardial infarction Bleifeld et al. ${ }^{4}$ found a negative correlation between infarct size calculated from serial CK determinations and left ventricular performance estimated from cardiac index and pulmonary end-diastolic pressure at right heart catheterisation.

In the present study of 101 patients infarct size calculated from blood enzyme levels showed a slight but insignificant negative correlation with physical capability, as measured by the duration of work at exercise soon after acute mycocardial infarction in the 53 patients (mean age $53.9 \pm 11 \cdot 1$ years) who were able to continue exercise until stopped by fatigue and dyspnoea. This correlation has the same tendency, but is not so pronounced, as the one which Paine et al. ${ }^{18}$ found in somewhat younger patients (mean $48 \pm$ 8 years) several months (median 4.5 , mean $13 \pm 4$ months) after acute myocardial infarction. As scar size angiocardiography has shown a very good correlation with infarct size calculated from serum CK MB levels, ${ }^{2}$ the reason for the quantitative differences between the two series might possibly be found in the different time span from acute infarction to the exercise test. Cardiac performance soon after acute infarction is presumably more uniformly depressed, and patients with a smaller infarct size are probably able to regain some of their physical capability in subsequent months.

In the present series systolic blood pressure and heart rate at rest were not related to infarct size, but the rise in systolic blood pressure during exercise showed a significantly negative correlation, and the increase in heart rate a significantly positive correlation, so that the rise in the pressure-rate product, and presumably the rise in myocardial oxygen consumption, remained unrelated to infarct size. The rise in systolic blood pressure during exercise seems to be lower in patients with larger infarcts, but a higher increase in heart rate compensates, thus bringing about the same myocardial oxygen consumption and cardiac performance.

Calculations of cardiac stroke volume from measurements of thoracic electrical impedance have shown a very good correlation with results from the dye dilution method $(\mathrm{r}=0.90)$, both at rest and at exercise. ${ }^{19}$

In the present series, the resting values of stroke volume and cardiac output, as calculated from impedance measurements, were essentially the same whether the infarcts were large or small, but the rise during exercise was to a significant extent negatively correlated with infarct size. Repeated measurements during the exercise test showed that stroke volume rose only at the lower levels of exercise, and that it remained unchanged at higher levels, the further rise in cardiac output being achieved entirely by an increase in heart rate. In some patients, both with large and small infarcts, the stroke volume did not increase at all during exercise; the total rise in cardiac output was achieved by an increase in heart rate. These findings tend to support the impression that treatment with a beta-blocking agent might not be suitable for patients with larger infarcts. When patients changed from a supine position to sitting on the bicycle, stroke volume and cardiac output fell considerably, nearly as much as the subsequent rise in stroke volume during exercise. It is not known, however, how much of this decrease reflects the wellknown orthostatic reduction in stroke volume, or how much might be changes in thoracic impedance simply because of the anatomical alterations from lying to sitting.

Depression of ST segments during exercise occurred in slightly over one-half of the patients with angina pectoris, and in two-fifths of those without angina pectoris, with no significant correlation with infarct size. Elevation of the ST segment, however, occurring in slightly less than one-third of the patients with and one-third of the patients without anginal pain showed a significantly positive correlation with infarct size. These observations are in accordance with earlier findings that exercise induced ST elevation is related to the extension of the akinetic myocardium after acute infarction. ${ }^{20}$

It is noteworthy that the $17 \%$ of the patients who experienced angina pectoris during exercise had significantly smaller infarcts, probably indicating an inverse relation between the extent of the infarcted and of the jeopardised myocardium. 
Previous studies ${ }^{21}$ have found that the occurrence of ventricular arrhythmias during the hospital phase of acute myocardial infarction was positively correlated to infarct size. The frequency of rhythm disturbances before and during exercise is much the same as that found in earlier series in our coronary care unit, 516 and by others using a symptom limited exercise test early after acute myocardial infarction; ${ }^{22}$ the frequency is somewhat higher than in series with preset limits for cardiac performance in this group of patients. ${ }^{23} 24$

In the present series, no significant correlation could be shown between infarct size and any kind of arrhythmia before or during exercise.

\section{References}

1 Opie LH. Myocardial infarct size. Part I. Basic considerations. Am Heart F 1980; 100: 355-72.

2 Rogers WJ, McDaniel HG, Smith LR, Mantle JA, Russell RO Jr, Rackley CE. Correlation of angiographic estimates of myocardial infarct size and accumulated release of creatine kinase $\mathrm{MB}$ isoenzyme in man. Circulation 1977; 56: 199-205.

3 Hori M, Inoue M, Fukui S, et al. Correlation of ejection fraction and infarct size estimated from the total CK release in patients with acute myocardial infarction. $\mathrm{Br}$ Heart F 1979; 41: 433-40.

4 Bleifeld W, Mathey D, Hanrath P, Buss H, Effert S. Infarct size estimated from serial serum creatine phosphokinase in relation to left ventricular hemodynamics. Circulation 1977; 55: 303-11.

5 Ibsen H, Kjøller E, Styperek J, Pedersen A. Routine exercise ECG three weeks after acute myocardial infarction. Acta Med Scand 1975; 198: 463-9.

6 Grande P, Hansen BF, Christiansen C, Næstoft J. Acute myocardial infarct size estimated by serum CK-MB measurements: clinical accuracy and prognostic relevance utilizing a practical modification of the isoenzyme approach. Am Heart $\mathcal{F}$ 1981; 101: 582-6.

7 Grande P, Christiansen C, Pedersen A, Christensen MS. Optimal diagnosis in acute myocardial infarction. A cost-effectiveness study. Circulation 1980; 61: 723-8.

8 Grande P, Christiansen C, Næstoft J. Creatine kinase isoenzyme MB assay by electrophoresis. Scand $\mathcal{f}$ Clin Lab Invest 1979; 39: 607-12.

9 Grande P, Hansen BF, Christiansen C, Næstoft J. Estimation of acute myocardial infarct size in man by serum CK-MB measurements. Circulation 1982; 65 (in press).

10 Kubicek WG, Karnegis JN, Patterson RP, Witsoe DA, Mattson RH. Development and evaluation of an impedance cardiac output system. Aerospace Med 1966; 37: 1208-12.

11 Shell WE, Sobel BE. Biochemical markers of ischemic injury. Circulation 1976; 53, suppl I: 98-106.
12 Geltman EM, Ehsani AA, Campbell MK, Schechtman $K$, Roberts $R$, Sobel BE. The influence of location and $C$ extent of myocardial infarction on long-term ventricular $\vec{F}$ dysrhythmia and mortality. Circulation 1979; 60: 805- क? 14.

13 Hossack KF, Bruce RA, Green B, Kusumi F, DeRouen 흘 TA, Trimble S. Maximal cardiac output during upright $\frac{\bar{s}}{\frac{}{T}}$ exercise: approximate normal standards and variations $\overparen{\mathbb{}}$ with coronary heart disease. Am f Cardiol 1980; 46: 204-12.

14 Epstein SE, Beiser GD, Stampfer M, Robinson BF, $\vec{O}$ Braunwald E. Characterization of the circulatory response to maximal upright exercise in normal subjects $\vec{\omega}$ and patients with heart disease. Circulation 1967; 35: $1049-62$

15 Davidson DM, DeBusk RF. Prognostic value of a single exercise test 3 weeks after uncomplicated myocardial.infarction. Circulation 1980; 61: 236-42.

16 Madsen EB, Rasmussen S, Svendsen TL. Multivariate long-term prognostic index from exercise ECG after $\mathrm{O}$ acute myocardial infarction. Eur $\mathcal{F}$ Cardiol 1980: 11:435-43.

17 Norris RM, Caughey DE, Deeming LW, Mercer CJ, , Scott PJ. Coronary prognostic index for predicting survival after recovery from acute myocardial infarction. Lancet 1970; ii: 485-8.

18 Paine TD, Dye LE, Roitman DI, et al. Relation of N graded exercise test findings after myocardial infarction to extent of coronary artery disease and left ventricularo dysfunction. Am $\mathcal{F}$ Cardiol 1978; 42: 716-23.

19 Denniston JC, Maher JT, Reeves JT, Cruz JC, Cymer-̄̄ man A, Grover RF. Measurement of cardiac output by气 electrical impedance at rest and during exercise. $\mathcal{F} A p p l_{\varrho}^{\circledR}$ Physiol 1976; 40: 91-5.

20 Dunn RF, Bailey IK, Uren R, Kelly DT. Exercise-음 induced ST-segment elevation. Correlation of thallium201 myocardial perfusion scanning and coronary arteriography. Circulation 1980; 61: 989-95.

21 Roberts R, Husain A, Ambos HD, Oliver GC, Cox JR $\mathrm{Jr}$, Sobel BE. Relation between infarct size and ventricular arrhythmia. Br Heart $\mathcal{F} 1975$; 37: 1169-75.

22 Sami M, Kraemer H, DeBusk RF. The prognostic significance of serial exercise testing after myocardiath infarction. Circulation 1979; 60: 1238-46.

23 Ericsson M, Granath A, Ohlsén P, Södermark T, Volpe U. Arrhythmias and symptoms during treadmill testing three weeks after myocardial infarction in 100 patients. I Br Heart F 1973; 35: 787-90.

24 Théroux P, Waters DD, Halphen C, Debaisieux JC Mizgala HF. Prognostic value of exercise testing soons after myocardial infarction. $N$ Engl f Med 1979; 301 N 341-5.

Requests for reprints to Dr Peer Grande, Cardiologie cal Laboratory, Glostrup Hospital, DK-2600 Glos울 trup, Denmark. 